\title{
La formación del director de coro desde una perspectiva decolonial
}

\author{
Gustavo Zgainer' y María Inés Burcet ${ }^{2}$ \\ gustavozgainer@hotmail.com \\ Laboratorio para el Estudio de la Experiencia Musical. Facultad de Artes. \\ Universidad Nacional de La Plata
}

\section{Resumen}

La carrera de Dirección Coral forma parte de la propuesta académica de diferentes universidades en Latinoamérica. En estos espacios de formación, los planes de estudio suelen poner el énfasis en el dominio y la comunicación de la gestualidad, la adquisición de técnicas de ensayo y ensamble, el conocimiento acerca de la correcta dicción idiomática, el estudio de la técnica vocal y la adquisición de habilidades auditivas. Sin embargo, estas capacidades muchas veces entran en conflicto con las necesidades y las exigencias que la actividad profesional demanda a los directores de coro, especialmente cuando las realidades de los grupos corales en los que los directores se desempeñan se alejan de las características propias de los grupos vocales profesionales. En este trabajo nos proponemos conocer algunas de las dimensiones de la realidad profesional con la cuales se encuentran los directores de coro al ejercer la profesión. Al mismo tiempo nos proponemos hacer un análisis crítico de la formación actual del director de coro desde una perspectiva decolonial con el fin de visibilizar los supuestos que subyacen a su formación y promover la necesidad de transformar esa realidad.

\section{Palabras clave}

dirección coral, formación profesional, coro vocacional, perspectiva decolonial. 


\title{
The formation of the choir director from a decolonial perspective
}

\begin{abstract}
The choral conducting career is part of the academic proposal of different universities in Latin America. In these training spaces, the study plans tend to emphasize the mastery and communication of gestures, the acquisition of rehearsal techniques, the knowledge about the correct idiomatic diction, the study of vocal technique and the acquisition of listening skills. However, these capacities often conflict with the needs and demands that the professional activity demands of choir conductors, especially when the realities of the choral groups in which the conductors perform are far from the characteristics of professional vocal groups. In this work we propose to know some of the dimensions of the professional reality that choir conductors encounter when practicing the profession. At the same time we propose to make a critical analysis of the current formation of the choir conductor from a decolonial perspective, in order to make visible the assumptions that underlie their formation and promote the need to transform that reality. Based on a set of interviews with choir conductors, analyzed using the Constant Comparison Method, we managed to find coincidences that led us to define some particular dimensions to continue thinking about institutional training, especially based on the needs that arise in the professional practice. We understand that it is increasingly urgent to be able to attend the context of professional practice and social demands as form of resistance against the coloniality of knowledge that imposes abstract content for abstract subjects.
\end{abstract}

\section{Key words}

choral conducting, professional training, amateur choir, decolonial perspective.

\section{Introducción}

La carrera de Dirección Coral forma parte de la propuesta académica de diferentes universidades en Latinoamérica. En estos espacios de formación, los planes de estudio suelen poner el énfasis en el dominio y la comunicación de la gestualidad, la adquisición de técnicas de ensayo y ensamble, el conocimiento acerca de la correcta dicción idiomática, el estudio de la técnica vocal y la adquisición de habilidades auditivas. El director de coro debe abordar durante su formación estrategias conceptuales, técnicas y gestuales vinculadas con las necesidades de un amplio repertorio musical que va del canto gregoriano a la música coral contemporánea.

Por lo general, se espera que el egresado de esta carrera pueda desempeñarse 
con solvencia en la dirección de coros polifónicos, especialmente en aquellos integrados por músicos profesionales con experiencia en la lectura de partituras y el desarrollo de una técnica vocal específica. Esto permitirá a los coreutas acceder a sus líneas vocales a partir de la lectura de la partitura, en algunos casos con apoyo de un asistente del coro, y durante los ensayos el trabajo con el director estará centrado en el abordaje interpretativo del repertorio.

El modelo de formación que se imparte en la mayoría de las instituciones universitarias que forman directores de coro, comprende muchas de las características que diferentes autores han asignado al denominado modelo conservatorio (Gainza, 2002; Kingsbury, 1988; Musumeci, 2002)

Este modelo de enseñanza musical surge en Europa como espacio de formación para los músicos de orquesta, a partir de la creación del Conservatorio de París (1795) y luego se extiende a toda América constituyéndose como paradigma único para la formación musical profesional. Lavignac (1950) describe la práctica de enseñanza de los conservatorios como dogmática y agrega que, en ese marco:

El profesor es considerado impecable e infalible, lo que diga debe ser aceptado como artículo de fe, y el ejemplo que da debe ser imitado servilmente. Si es cantante o instrumentista, enseña a sus alumnos a tocar o cantar como él, a respirar, a pronunciar, a sostener el arco como él; les comunica algo de su propio estilo, los forma a su imagen, tan bien que, cuando se los escucha, se puede decir sin vacilar: éste es alumno de tal profesor. Es lo que se llama formar escuela. [...] Cuando el maestro es realmente un gran artista, sabe dejar a cada uno lo que le es necesario para constituir su individualidad, y se consagra especialmente a desarrollar las cualidades innatas. (Lavignac, 1950, p. 366).

El modelo conservatorio ha sido caracterizado por su énfasis en los siguientes aspectos: (i) la notación musical como único modo de acceso a la música, donde la partitura se concibe como representación completa de la misma; (ii) la ejercitación técnico instrumental, donde el aprendizaje gira en torno al abordaje del repertorio desde una técnica específica; (iii) el acceso al repertorio académico de la música europea como repertorio privilegiado para el desarrollo musical; (iv) el encuadre diádico centrado en la relación maestro-alumno, es decir sustentado en la idea de que hay un sujeto que conoce y otro que no conoce; y (v) la valoración de los desempeños individuales los cuales se fundan en el estudio solitario (Gonnet, 2015; Holguín y Shifres, 2015; Kingsbury, 1988; Musumeci, 2002).

Estas características perviven en los modelos de enseñanza musical más allá de la institución que le dio origen. Podemos encontrar situaciones de enseñanza similares a la descripta arriba que se producen por fuera de los conservatorios, como ocurre en numerosas clases particulares de instrumento o canto, y también 
podemos encontrar los principales rasgos de este modelo en la concepción que subyace a la educación universitaria, en este caso en la formación del director de coro.

Estos rasgos se advierten, por ejemplo, cuando se concibe al coro profesional polifónico como único destinatario de la práctica profesional, se centraliza en el repertorio de música académica europea, se parte del establecimiento de una relación asimétrica entre director y coro (donde el primero es quien toma todas las decisiones) o se sobrevalora el desempeño individual como previo y constitutivo para la práctica grupal (que queda en evidencia, por ejemplo cuando se espera que cada coreuta aprenda su parte como requisito previo para la concertación grupal).

La formación del director de coro deja de lado la posibilidad de dar lugar a otras formas de concebir las agrupaciones corales, incluso las que podrían poner en tensión su propio rol, en tanto único líder.

Para dar un ejemplo, en la ciudad La Plata (ciudad capital de la Provincia de Buenos Aires, Argentina), en donde residimos ambos autores de este trabajo, actualmente existen cerca de un centenar de coros, entre los cuales sólo una decena tienen las características de un coro profesional. Por el contrario, en la mayoría de los coros habita una gran heterogeneidad de situaciones con relación a: (i) las expectativas y búsquedas de los coreutas, que van desde encontrar un espacio que los motive a cantar o conocer música hasta la posibilidad de encontrar un espacio para establecer relaciones sociales; (ii) la diversidad en la participación de los integrantes del coro, quienes en algunos casos se sienten motivados a cantar, pero en muchos otros siente inseguridad; (iii) la conformación del grupo, donde por lo general resulta poco posible equilibrar la cantidad de voces femeninas y masculinas, mantener un número estable de integrantes o incluso sus integrantes cambian permanentemente; (iv) el modo en que utilizan una partitura, donde, como señalan Beltramone y Burcet (2019) encontramos que la misma podía ser utilizada a partir de diferentes aproximaciones como seguir el texto, localizar las entradas, seguir el contorno melódico, entre otras posibilidades, las cuales no implican necesariamente las estrategias tradicionales de lectura de una partitura (nota por nota, por ejemplo).

Esta diversidad no es considerada en la formación universitaria del director de coro y entendemos que esto se debe principalmente a dos motivos. En primer lugar, porque se entiende que el destinatario único de las prácticas profesionales del director de coro será siempre el coro profesional polifónico y, en segundo lugar, porque se considera que, si el director puede desempeñarse en un coro profesional polifónico, entonces estará preparado para abordar las realidades particulares de otras agrupaciones corales por ser éstas últimas más simples, incluso dando por sentado que requerirían las mismas habilidades. 
Por lo tanto, en este trabajo nos proponemos conocer algunas de las dimensiones de la realidad profesional con la cuales se encuentran los directores de coro al ejercer la profesión. Al mismo tiempo nos proponemos hacer un análisis crítico de la formación actual desde una perspectiva decolonial, con el fin de visibilizar los supuestos que subyacen a la formación del director de coro y promover la necesidad de transformar esos espacios de formación profesional.

Para contextualizar el trabajo nos proponemos repasar y poner de relieve algunas dimensiones del problema que resultarán claves para su análisis.

\section{Fundamentación}

Aníbal Quijano (1992) propuso el concepto colonialidad para referirse a las formas y los efectos del proceso de colonización, en particular a la constitución de la interioridad misma del imaginario del colonizado y de su propia subjetividad. El autor explica que la cultura europea no sólo se impuso sobre otras culturas no europeas, sino que además se convirtió en una aspiración ya que la misma otorgaba cierta ilusión de acceso al poder y "después de todo, más allá de la represión, el instrumento principal de todo poder es su seducción" (Quijano, 1992, p. 439).

La colonialidad a la cual refiere Quijano (1992) se concretó, entre otras formas, a través de la colonialidad del saber (Castro-Gómez, 2007; Lander, 2000; Quijano, 2002; Walsh, 2007), entendida como el proceso a través del cual se construyó y consolidó una forma de pensamiento y conocimiento que se universalizó en las sociedades coloniales. De este modo, las formas de conocimiento europeas se convirtieron en las únicas válidas, objetivas y universales (Lander, 2000), lo que condujo al mismo tiempo a subalternizar, folclorizar o invisibilizar otras representaciones y saberes que quedaron relegados a objetos de menor valor, silenciados o sin poder de enunciación.

Como señala Lander (2000), una de las consecuencias más importantes de la colonialidad del saber fue la creación de instituciones de enseñanza como dispositivos para permitir y mantener el control y la legitimidad del conocimiento. En tal sentido, Wallerstein expresa "Las universidades han sido a la vez los talleres de la ideología y los templos de la fe" ([1983] 2006, p. 72). Universidades y conservatorios, en el caso de la enseñanza de la música, han funcionado como defensores del saber eurocéntrico, incluso impidiendo que otras visiones diferentes del mundo alcanzaran legitimidad. Como señalan Baquero, Caicedo y Rico (2015), las instituciones modernas "son jueces que se encargan de descalificar todo lo que está más allá de sus métodos como algo mítico o provincialista" (pp. 79-80)

En la experiencia musical, la colonialidad del saber muchas veces queda en evidencia en expresiones de sentido común o en aquello que se asume como ver- 
dad única. Por ejemplo, cuando se entiende que hay un "saber cantar" que implica hacerlo sólo a partir de una técnica específica. En tal sentido, en una encuesta realizada hace unos años en el ingreso a las carreras de música de una universidad, solamente el $9 \%$ de los estudiantes declaró que cantaba, en respuesta una pregunta acerca de las modalidades a través de las cuales hacían música ${ }^{3}$. Lo que llevó a considerar, entre diferentes argumentos, que los estudiantes no visualizaban sus propias prácticas de canto porque el ámbito universitario los inhibiría para adjudicarles un valor heurístico tanto a sus propios repertorios como a las diferentes modalidades de emisión vocal y los contextos sociales en los que las mismas podían tener lugar. El imaginario alrededor de qué es aquello a lo cual la Universidad considera como cantar, podría favorecer esa desvalorización, entendiendo que en los ámbitos de enseñanza formal de la música, la experticia implica tanto la adquisición de una técnica específica (por ejemplo el canto lírico) a la cual se accede mediante la práctica sostenida, el dominio de la lectura en el pentagrama y el conocimiento de un determinado repertorio académico (de origen europeo) (Shifres y Burcet, 2016). En síntesis, los estudiantes no identificarían sus formas de cantar como las propias de un saber y entonces se excluirían del saber mismo.

Otra situación que permite observar el modo en que la colonialidad cultural ha perfilado nuestra subjetividad se advierte en ciertas expresiones que con habitualidad producen quienes han aprendido a tocar un instrumento a partir de la imitación o "de oído" y entonces, aun cuando tienen experiencia en la práctica de ejecución instrumental, incluyendo prácticas de conjunto, se refieren a sus conocimientos advirtiendo "toco hace muchos años, pero no sé música". Asumiendo que "saber música" implica haber aprendido en una institución especialmente dedicada a la enseñanza y vinculada con ciertos repertorios y técnicas de ejecución específicas.

Estos son sólo algunos ejemplos que muestran aspectos más superficiales y visibles del problema. Entendemos que la colonialidad del saber opera a partir de tramas más profundas, controlando la subjetividad y occidentalizando el imaginario, de modo que por lo general sus formas no se advierten con facilidad dado que sus supuestos se instalan como verdades únicas y universales.

En el campo de la música, diferentes estudios decoloniales realizados en la última década se han propuesto el desafío de encontrar nuevos marcos epistemológicos y ontológicos para repensar la formación musical y han desarrollado algunas alternativas al pensamiento eurocéntrico-colonial en el campo de la investigación musical. Algunos estudios promueven la necesidad de considerar la experiencia colectiva como condición de posibilidad para el hacer musical, poniendo en valor las instancias colaborativas y de aprendizaje entre pares (Gonnet, 2015); también se propuso la necesidad de involucrar el cuerpo, el movimiento, el entorno físico y social en la actividad de escuchar y pensar la música para establecer puentes entre 
la escucha musical musicológica y la naturaleza afectiva de la actividad musical (Holguín y Shifres, 2015; Shifres y Gonnet, 2015); se propuso promover la elaboración de categorías diferentes a las de la notación musical las cuales incorporaren descripciones del vínculo del hombre con la naturaleza (Holguín y Shifres, 2015; Shifres y Gonnet, 2015), y también se propuso una perspectiva decolonial para pensar la notación musical a partir de la posibilidad de concebirla como un sistema de representación (Burcet, 2017).

En este trabajo nos proponemos reflexionar sobre la formación académica del director de coro, que es un espacio en donde persiste una profundamente perspectiva eurocéntrica. Entendemos que, como en muchos otros espacios de formación universitaria, el desafío más profundo es el de deconstruir el carácter universal y natural de las ontologías y epistemologías que allí se asumen. Es por ello que nos proponemos estudiar las prácticas epistémicas que tienen lugar en la cotidianeidad del trabajo del director de coro a partir de las problemáticas que conviven en su campo de acción y que suelen estar invisibilizadas dentro de modos canónicos del conocimiento profesional porque no se reconocen en ellas los rasgos de institucionalización propios de las modalidades de aprendizaje validadas por los modelos dominantes.

\section{Método}

El análisis de los datos del presente estudio se basa en la metodología de la Teoría Fundamentada inicialmente propuesta por Glaser y Strauss (1967) y posteriormente ampliada y desarrollada por Strauss y Corbin (1990). El enfoque metodológico conduce a desarrollar teoría cimentada en información sistemáticamente recogida y analizada (Strauss y Corbin, 1994). Esta metodología ha sido especialmente utilizada en la sociología para el análisis de datos de texto extensos y utiliza dos estrategias metodológicas: el Método de Comparación Constante (MCC) y el Muestreo Teórico (MT). El Método de Comparación Constante propone un conjunto sistemático de procedimientos de comparación y codificación de datos, mientras que el Muestreo Teórico corresponde a la particularidad en que la muestra es conformada. La metodología resulta apropiada para identificar las variables que inciden en un problema cuando las mismas no se conocen de ante mano, como es el caso del presente de estudio.

En una primera instancia se comparan los datos obtenidos, en cada sujeto y entre sujetos, se buscan similitudes y diferencias, otorgándole las mismas denominaciones a aquellos datos que comparten una misma idea. Luego los datos son categorizados y/o clasificados en categorías. En una segunda instancia, se buscan aspectos recurrentes con el fin de definir y ejemplificar las propiedades de cada 
una de las categorías, para finalmente dar lugar a una teoría fundamentada en los datos la cual da respuesta a la pregunta inicial de investigación.

Es importante destacar que la teoría que se origina en los datos no es una teoría general sino, más bien, una teoría de alcance intermedio (middle-range theories), en términos de Merton (1968), es decir, un conjunto de enunciados que proponen una serie se supuestos de alcance limitado y que permiten describir los datos en el contexto en que se dan y a la luz de la pregunta de investigación propuesta. De este modo la teoría permitirá comprender, explicar el fenómeno que es objeto de estudio. La potencialidad heurística de las categorías esbozadas será mostrada a partir del estudio de casos nuevos, los cuales deberán quedar incluidos en las categorías propuestas y no derivar en categorías adicionales.

\section{Instrumento de indagación}

Para el relevamiento de datos se utilizó la entrevista como herramienta para acceder a las concepciones y perspectivas propias de los directores de coro. Estas entrevistas buscaban descubrir los problemas del ejercicio de su profesión para visibilizar aspectos no contemplados en la formación académica.

Desde esta perspectiva resulta interesante estudiar las prácticas epistémicas que tienen lugar en la cotidianeidad del trabajo del director de coro a partir de las problemáticas que conviven en su campo de acción y que suelen estar invisibilizadas dentro de modos canónicos del conocimiento profesional. Esta invisibilización se debe a que no se reconocen en ellas rasgos de institucionalización que caracterizan a las modalidades de aprendizaje validados por los modelos dominantes.

Para ello se realizaron preguntas abiertas tendientes a recabar información acerca de las características de los coros en los que se desempeñan, la metodología de los ensayos, los criterios bajos los cuales eligen el repertorio, los problemas que encuentran en el ejercicio de la práctica profesional y la relación que pueden establecer entre las necesidades de que les demanda el ejercicio profesional y las características de la formación universitaria.

\section{Descripción de la muestra}

Siguiendo el Muestreo Teórico (MT), a partir del cual la muestra se ajusta a los requerimientos que el análisis demanda, y dado que a partir de la décima entrevista observamos que las problemáticas señaladas por los directores eran recurrentes, finalmente la muestra quedó definida en diez casos.

Se entrevistaron entonces individualmente a diez directores de coro, todos ellos egresados de la universidad, quienes al momento de ser entrevistados se encontraban dirigiendo dos o más coros. Todas las entrevistas fueron grabadas. 


\section{Resultados y discusión}

Siguiendo el Método de Comparación Constante (MCC), el análisis de datos se desarrolló en tres etapas. En una primera etapa se analizaron y compararon los datos buscando similitudes y diferencias entre ellos. Se otorgó una misma denominación a aquellos datos que compartían una misma idea o dimensión del problema y se codificaron partes de las entrevistas de acuerdo con esas categorías. Para esta primera etapa se utilizó el programa NVIVO10, el cual permitió reunir las referencias durante el proceso de categorización.

En una segunda etapa se discutieron y delimitaron las categorías identificadas, su alcance, propiedades y características. En esta etapa se seleccionaron aquellas categorías que resultaban más adecuadas a los objetivos del presente estudio.

En una tercera etapa, se procuró definir las particularidades de cada una de las categorías establecidas. Para ejemplificarlas, a partir de las referencias marcadas en los textos, se seleccionaron aquellas que mejor permitían dar cuenta el alcance de la categoría en cuestión.

Y finalmente, las categorías fueron organizadas en dos grupos. Por un lado, aquellas que evidenciaban ciertas problemáticas del enfoque actual, todas ellas vinculadas con el marco teórico, es decir con la persistencia de ciertos rasgos de colonialidad en la educación en general y de la formación del director de coro en particular; y por otro lado, aquellas categorías que se ponían en evidencia a partir de las necesidades que manifestaban los directores con relación a la posibilidad de ejercer su profesión en el contexto de su realidad profesional situada.

A continuación, se desarrolla cada una de las categorías, las mismas se presentan en tanto oposición de perspectivas acerca de problemáticas vinculadas: por un lado, la identificación de rasgos de colonialidad en la formación actual y por el otro la posibilidad de enfocar esa dimensión hacia una mirada que trascienda ese enfoque.

\section{Del monitoreo de la práctica en la clase al monitoreo en el contexto real}

Uno de los primeros aspectos que advertimos en los testimonios de los directores entrevistados es la brecha existente entre el contexto en el que se producen las prácticas de dirección coral durante la formación universitaria y la realidad del ejercicio profesional. Esta relación permite señalar algunas dimensiones que resultan claves para comprender la concepción eurocéntrica que sustenta la formación académica del director de coro.

Los entrevistados coinciden en señalar que, durante su formación, la práctica de dirección suele desarrollarse en el contexto del aula donde son los mismos 
estudiantes de la carrera quienes asumen el rol de coreutas. En este sentido Pablo expresa "cuando estudiamos obras en la facultad lo hacemos con cantantes formados, cuando estudiamos técnica vocal lo hacemos sobre nosotros mismos entonces luego falta poder trabajar todo esto en el otro, en el coreuta".

Los entrevistados destacan algunas ventajas de esta modalidad afirmando que generalmente facilita el armado de las piezas que se quieren interpretar y el abordaje de los contenidos, ya que se trata de dirigir a los propios compañeros quienes, entre otras cuestiones, conocen el contenido acerca del cual se trabaja. La modalidad permite que, durante la práctica, el acento esté puesto en las correcciones y ajustes del gesto del director y en la relación que se establece entre estas adecuaciones y el resultado musical. Sin embargo, todos los entrevistados advierten que la modalidad presenta importantes limitaciones al momento de interactuar con un verdadero coro, ya que la permanente simplificación del contexto de práctica finalmente deriva en una limitación de las estrategias de quienes se están formando. En tal sentido Julián expresa: "En la universidad tus compañeros ya saben qué hay que hacer, qué se espera que ellos hagan y entonces no hay lugar para imprevistos, todos parecen hacerte el juego y vos crees que está todo controlado".

Los entrevistados hacen referencia a variadas problemáticas que surgen en los contextos reales, vinculadas con la elección y adecuación del repertorio, las características del espacio físico, la dinámica de ensayo, las relaciones entre los integrantes del grupo, etc. En este sentido Emilia comenta:

"Cuando trabajás con niños, por ejemplo, tenés que tener en cuenta muchísimas cosas relacionadas con la seguridad, conocer la ubicación de los baños, hacer un uso adecuado de las gradas, tramitar autorizaciones de los padres, esperar a que retiren a todos los niños al finalizar el ensayo y hasta mirar los enchufes del lugar".

El problema aquí parece residir en el desagregado que se hace de la práctica profesional al diseñar estrategias de dirección más allá de las particularidades de los grupos. Esta separación no es casual, implica un modo de concebir el ejercicio de la profesión que niega y excluye al sujeto al cual se dirige esa práctica. De este modo la práctica de dirección es abordada como universal, como una práctica que puede desarrollarse con independencia de un contexto específico, cuando por el contrario consideramos que el mayor desafío del director reside en la posibilidad de redefinir y adecuar su desempeño según las particularidades de los variados grupos humanos en los cuales desarrolla su actividad. En este sentido, a partir de su experiencia en dirigir diferentes coros, Héctor expresa:

"me di cuenta de que cada grupo tiene una identidad y una búsqueda distinta, aunque sea con un mismo director y una misma forma de trabajo, porque la entrega 
que yo le brindo a cada coro siempre es total pero el resultado de cada grupo, por sus cualidades individuales y colectivas, siempre es diferente".

Con relación a este punto, entendemos que las instituciones de formación deberían promover espacios de práctica en coros y agrupaciones vocales con diversidad de recorridos y posibilidades. Siguiendo la idea que se desarrolla en el campo de la Educación Musical donde el formador de formadores asiste al contexto mismo donde el docente dicta sus prácticas, de manera similar, durante la formación académica, el estudiante debería realizar sus prácticas en agrupaciones corales provenientes de diferentes instituciones (escuelas, clubes, bibliotecas, iglesias, hospitales, etc), y con diversos grupos etarios (de niños a personas adultas mayores, incluyendo personas con discapacidades). De este modo, durante la formación podrían abordarse diferentes problemáticas tales como analizar qué repertorio resulta más adecuado para cada grupo, cuáles estrategias pueden desplegarse, cómo organizar el tiempo de ensayo, qué alternativas plantear respecto a las características del espacio, cuáles recaudos considerar, cómo favorecer la motivación del grupo, entre otras.

Por supuesto que no se trata simplemente de un conjunto de estrategias pedagógicas, se trata de asumir un posicionamiento epistemológico diferente con relación a los alcances de la actividad profesional del director de coro. Si se concibe la actividad profesional como un conjunto de conocimientos técnicos y teóricos, entonces la práctica se desarrollará con independencia de los sujetos a quienes ese conocimiento va dirigido (o tal vez suponiendo un sujeto ideal), pero si concebimos la actividad coral como una práctica social y situada, el problema se plantea en términos completamente diferentes. Aunque se adquiera un conjunto de gestos, estrategias y repertorios, estos deberán siempre redefinirse según cada contexto contemplando y abriendo las posibilidades al significado, la identidad y la comunidad en donde esas prácticas tengan lugar.

\section{De la dirección del coro polifónico profesional al armado del coro vo- cacional}

La formación académica del director de coro está orientada hacia un tipo particular de agrupamiento: el coro polifónico profesional. En estos coros, los integrantes suelen tener conocimiento de la notación musical, desarrollo de una determinada técnica vocal, experiencia en cantar piezas polifónicas, familiaridad con el repertorio de gestos que utilizan los directores, entre otras habilidades. A su vez, en tanto agrupación suelen presentar una distribución equilibrada de integrantes por cuerda, cierta frecuencia y modalidad de ensayos, presentaciones pautadas, instituciones que los financian, etc. Los recursos y estrategias que desarrollan los directores de coro en las universidades suelen estar orientados especialmente (y 
por lo general, únicamente) a este tipo de agrupaciones vocales que tienen su origen en las sociedades europeas. En tal sentido, Marcos señala: "nosotros nos formamos en la dirección musical de obras más que nada académicas, voces preparadas de gente que canta y de gente que lee, pero después la realidad profesional es muy diferente, son muy pocos los coros que tienen estas características".

Todos los entrevistados señalan que hay una marcada diferencia entre el coro profesional polifónico y las agrupaciones corales vocacionales en las que más frecuentemente los directores desarrollan su profesión. En este sentido, destacan que las habilidades que se requieren para abordar la práctica profesional en los coros vocacionales, no son ni más ni menos complejas, sino que son diferentes.

En un coro vocacional, los integrantes se acercan a participar motivados por variados intereses. En algunos casos porque les gusta cantar y disfrutan de hacerlo, en otros porque buscan un espacio donde establecer vínculos sociales, a veces porque quieren desarrollar sus posibilidades vocales y otras veces porque buscan un espacio de esparcimiento. Los recorridos también son muy variados con relación a la experiencia en la práctica vocal o en el desempeño en la lectura de partituras.

Habitualmente estas agrupaciones vocales surgen de la propia iniciativa del director, quien acerca la propuesta a una institución que puede ser un club, una iglesia, un centro de jubilados, un colegio profesional, una escuela, entre otros. La propuesta muchas veces no tiene antecedentes en la misma institución y con relación a esto Leandro explica: "en general las autoridades del lugar no saben lo que es un coro y cómo se trabaja, mucha gente lo ve como una actividad menor, entonces hay que contarles de que se trata, donde estudiaste para hacer lo que vas a hacer. Hay que enseñarles"

Una vez aprobado el proyecto se abre una convocatoria abierta que es impulsada por el propio director y la institución. A partir de allí, los entrevistados describen múltiples dificultades e imprevistos que surgen durante un proyecto de estas características, como por ejemplo la adecuación del espacio, el acceso a los materiales de ensayo (instrumento, partituras), el acompañamiento de la institución y hasta los honorarios del mismo director.

Julián plantea que en el inicio todo es impredecible:

"en el momento tenés que ver cómo hacer y qué cantar, porque participan muchas personas con expectativas y formas de cantar bien distintas. Y siempre es preferible poder cantar en esos grupos iniciales una canción al unísono simple, afinada, que fluya y que los coreutas lo disfruten".

Entre las problemáticas propias de los coros vocacionales, los entrevistados 
mencionan que suelen ser grupos muy heterogéneos en los cuales hay integrantes que no conocen su propia voz y tampoco su registro vocal o que les da vergüenza cantar; coros donde suelen haber muchas mujeres y pocos hombres, o personas con edades muy diferentes; grupos que requieren la necesidad de adaptar los arreglos incluso durante el mismo armado de la pieza, ya sea porque encuentran dificultades o porque los integrantes no permanecen, etc.

Otro aspecto que señalan los directores es la constante preocupación por la retención de los integrantes y por la ampliación y la permanencia del grupo. Los directores mencionan diferentes estrategias tendientes a motivar a los coreutas: viajes, reuniones sociales y una cantidad mínima de presentaciones son mencionados por todos ellos.

Finalmente todos los directores entrevistados coinciden en destacar que la formación debería contemplar el abordaje de diversas problemáticas vinculadas con los grupos vocacionales. Algunas problemáticas propias del trabajo con el grupo, por ejemplo conocer diferentes dinámicas de ensayo, analizar estrategias motivacionales y de formación vocal; otras relacionadas con el armado del proyecto mismo como por ejemplo, poder fundamentar la necesidad de que una institución tenga un coro, aspectos legales con relación a la música y las grabaciones, entre otras.

Como señalamos en la introducción de este trabajo, habitualmente en los espacios académicos se entiende que, si el director puede desempeñarse en un coro profesional polifónico, entonces estará preparado para abordar las realidades particulares de otras agrupaciones corales por ser éstas últimas más simples, incluso dando por sentado que requerirían las mismas habilidades. Esta perspectiva está profundamente arraigada en las instituciones e implica una concepción eurocéntrica que menosprecia ciertas prácticas, relegándolas y subalternizándolas. Es un ejemplo del modo en que las instituciones académicas promueven las formas del saber eurocéntrico impidiendo que otras formas de conocer y de hacer, alcancen la misma legitimidad.

\section{Del énfasis en el tecnicismo a la formación integral del director}

Los entrevistados advierten que un aspecto fundamental que no es considerado durante la formación profesional del director de coro es el que hace referencia a las relaciones interpersonales. El vínculo que se establece entre el director y los coreutas suele quedar librado a la voluntad de cada uno, tal vez porque se considera que corresponde a la vida privada de las personas.

Con relación a este punto, Julio expresa: "Hoy en día, después de doce años de haberme recibido me doy cuenta de que la cuestión humana, el trato con las personas y los grupos son lo más importante". Y en el mismo sentido, Héctor 
destaca que "es necesario aprender a conocer al otro, escucharlo, ver que necesita, que quiere, que le gusta o que no, y eso también va ayudando no solo al buen manejo del grupo sino también a elegir el repertorio adecuado, la modalidad de los ensayos, entre otras tantas cosas".

Además, todos los directores señalan la necesidad de intervenir en diferentes oportunidades resolviendo diversas situaciones que surgen al interior de los coros. Por ejemplo, Silvina explica: "Hasta ahora nunca trabajé en un grupo en el cual yo no tenga que atender a algún aspecto motivacional o humano, o de grupo, o psicológico" En este sentido, los directores advierten sobre la necesidad de contar con herramientas para poder abordar estas problemáticas.

Otro aspecto que surge de las entrevistas es el de la autoridad y la jerarquía. La formación profesional le confiere al director una fuerte legitimidad. El director es quien habla en nombre de la Música y su lugar se asume como incuestionable. Sin embargo, los entrevistados señalan que estas asimetrías de poder, que también tienen una raigambre colonial ya que se asume que es sólo el director quien tiene el conocimiento, se han ido modificando progresivamente a través del tiempo. Todos ellos mencionan diferentes situaciones que demuestran que los coros se han vuelto espacios cada vez más democráticos y no sólo para la elección del repertorio. Julián explica que suele compartir conversaciones con todo el grupo para escuchar y atender a sus necesidades y expresa: "cada tanto hago una charla entre todos, una puesta en común, en la que todos hablamos un poco sobre la experiencia con en grupo, me interesa saber lo que sienten, conocer qué cosas mejorarín o cambiarían, lo que sacarían y lo que agregarían. Aunque te jugás un poco a que te cuestionen muchas cosas siempre me resulta muy buena y positiva la charla".

Los directores señalan que la formación profesional debería contemplar espacios para poder discutir sobre los vínculos que el director establece con los coreutas, analizar el alcance y las posibilidades de los roles y las jerarquías, abordar el estudio de las relaciones interpersonales que se establecen en torno al grupo de trabajo, por supuesto todo esto asumiendo a los coreutas como sujetos de conocimiento.

\section{Del repertorio impuesto al repertorio consensuado}

Durante la formación institucional las estrategias de dirección han estado siempre orientadas a la música académica. El estudiante transita su formación abordando piezas de los diferentes períodos de la historia de la música europea y sólo se incluye una reducida selección de piezas de autores latinoamericanos y música popular.

En sus testimonios, los directores señalan que encuentran una importante falencia entre el repertorio que se aborda en la universidad y las necesidades de los 
variados grupos en los que se desempeñan. Las limitaciones que encuentran se deben especialmente al hecho de que tanto la conformación de los grupos como los intereses resultan muy diferentes a los propios y particulares de los coros polifónicos profesionales. En este sentido Emiliano expresa:

"cuando salís de la facultad y encontrás gente que nunca cantó en coro ¿cómo haces? Si vas con el repertorio de música académica de la facultad no vas a poder armar nada. Por eso creo que en la formación debería haber un acercamiento más profundo a la música coral popular, porque si bien hay una asignatura de música popular, se trabaja muy poco repertorio sobre música popular coral"

Los directores destacan que el repertorio suele ser un tema muy sensible en los grupos y que lejos de tratarse de una decisión del director, la elección surge siempre a partir de consensos. En el relato de Rodrigo se pone en evidencia el lugar central que tienen el repertorio para la conformación misma del coro:

"Me dijeron que ahí habían tenido coros, pero ninguno funcionó porque ellos son andaluces, les gusta su música, y han venido muchos directores a tratar de hacer música antigua y cosas que a la gente del círculo no les gusta cantar, entonces pregunte que les gustaría cantar, me dijeron que coros rocieros, les pedí grabaciones (porque en esa época no había internet), y sin tener ni la menor idea de ese tipo de música arme un coro rociero, sacando los temas de oído hacia los arreglos, así empecé, haciendo un coro rociero que hoy tiene dieciocho años"

Todos los entrevistados insisten en señalar que es el director quien tiene que adaptarse a los intereses del coro y no el coro a los deseos musicales del director. En este sentido, los entrevistados coinciden en expresar que la mejor manera de pautar el repertorio es inicialmente evaluar los intereses y posibilidades musicales del grupo y realizar propuestas a partir de ello, considerando el contexto social, las edades, la institución entre otros aspectos. Así es que Mariano comenta: "Cuando comienza el año hacemos una lista de las canciones que nos gustaría cantar (siempre teniendo en cuenta las posibilidades técnicas), y teniendo en cuenta que les guste más a ellos que a mí, porque son ellos los que las van a cantar y entonces hago el arreglo".

De este modo, la formación del director debería promover estrategias para abordar un repertorio mucho más amplio como así también la posibilidad de hacer diferentes tipos de arreglos. Tal vez la lógica de organizar el repertorio de dirección alrededor de los períodos históricos de la música europea ya no sea el criterio más adecuado. 


\section{Conclusiones}

En este trabajo nos propusimos conocer las problemáticas particulares que tienen lugar en el trabajo profesional del director de coro especialmente en quienes ejercen la actividad en coros vocacionales y fueron formados en la universidad. Analizamos los testimonios de los directores a partir de algunos conceptos claves de la perspectiva decolonial aplicando una metodología cualitativa, definimos un conjunto de categorías donde pudimos identificar ciertos rasgos de colonialismo que subyacen a muchas de las decisiones vinculadas con la formación del director de coro y planteamos posibles alternativas.

Durante las entrevistas los directores señalaron diferentes falencias e incomodidades que fueron encontrando y siguen encontrando al llevar los conocimientos adquiridos en la universidad a los contextos en que desarrollan su actividad profesional. Estas falencias e incomodidades fueron dejando al descubierto diferentes dimensiones propias de las concepciones epistemológicas que subyacen al perfil del director de coro. Un perfil que parecerían responder a las necesidades de las sociedades europeas del siglo XVIII o XIX antes que a las necesidades propias y actuales de las sociedades latinoamericanas.

Esto permitió advertir el modo en que la complicidad con la colonialidad del poder continúa persistiendo en muchas instituciones de enseñanza de la música y en particular en este contexto. En tal sentido, señalamos la necesidad urgente de incorporar en la formación universitaria otras formas y maneras de pensar y sentir la actividad coral más allá del occidentalismo. En particular, las alternativas que se plantearon en este trabajo estarían orientadas a: (i) concebir la actividad coral como una práctica social y situada que debe producirse y analizarse siempre en contexto (ii) contemplar el abordaje de diversas problemáticas vinculadas con los grupos vocacionales, (iii) considerar el análisis y el estudio de las relaciones interpersonales y las dinámicas de grupo; y (iv) promover estrategias tendientes a ampliar y a adecuar el repertorio y las estrategias para su elección.

Entendemos esta posibilidad como un modo de resistencia contra la colonialidad del saber que impone contenidos abstractos para sujetos abstractos. Pero, además entendemos la urgencia de esta alternativa para poder seguir impulsando esa fuerza transformadora que representa la universidad en el campo del conocimiento. 
Epistemus - volumen 8 - número 2 (Diciembre de 2020)

DOI: https://doi.org/10.24215/18530494e 023

\section{Notas}

1. Gustavo Zgainer: https://orcid.org/0000-0002-6959-6012

2. María Ines Burcet: https://orcid.org/0000-0003-2969-7916

3. Encuesta realizada a comienzos de 2015 en la Facultad de Bellas Artes - Universidad Nacional de La Plata. Datos de circulación interna, inéditos.

\section{Referencias}

Baquero, S.; Caicedo J. y Rico, J. (2015). Colonialidad del saber y ciencias sociales: una metodología para aprehender los imaginarios colonizados. Análisis Político (85), pp. 76-92. https://doi.org/10.15446/anpol.v28n85.56248

Beltramone, C. y Burcet, M.I. (2019). El rol de la partitura en el coro amateur. En N. Alessandroni; B. Torres Gallardo y C. Beltramone, C. (Eds.), Vocalidades: la voz humana desde la interdisciplina. (pp. 417-442). La Plata: GITeV. Disponible en: http://sedici.unlp.edu. ar/handle/10915/89641

Burcet, M.I. (2017). Hacia una epistemología decolonial de la notación musical. Revista Internacional de Educación Musical, 5, 129-138. https://doi.org/10.12967/RIEM-20175-p129-138

Castro Gómez, S. (2007). Decolonizar la universidad. La hybris del punto cero y el diálogo de saberes. En S. Castro-Gómez y R. Grosfoguel (Eds.), El giro decolonial. Reflexiones para una diversidad epistémica más allá del capitalismo global (pp. 79-91). Bogotá: Iesco-Pensar-Siglo del Hombre Editores.

Gainza, V. d. (2002). Pedagogía musical. Dos décadas de pensamiento y acción educativa. Buenos Aires: Lumen.

Glaser, B.G. y Strauss, A.L. (1967). The discovery of Grounded Theory. Strategies for qualitative research. Chicago: Aldine.

Gonnet, H. D. (2015). Aprendiendo música en el encuentro. La construcción de conocimientos musicales a través de la práctica comunitaria y situada. Tesis de Maestría, inédita. Facultad Latinoamericana de Ciencias Sociales (FLACSO) y Universidad Autónoma de Madrid (UA). https://doi.org/10.35537/10915/58893

Holguín Tovar, P. J. y Shifres, F. (2015). Escuchar música al sur del rio Bravo. Desarrollo y formación del oído musical desde una perspectiva latinoamericana. Calle 14, 10 (15), 40-53. https://doi.org/10.14483/udistrital.jour.c14.2015.1.a04 
Kingsbury, H. (1988). Music, talent, and performance: A conservatory cultural system. Philadelphia: Temple University Press.

Lander, E. (Ed.) (2000). La colonialidad del saber: eurocentrismo y ciencias sociales. Perspectivas latinoamericanas. Buenos Aires: CLACSO.

Lavignac, A. (1950). La educación musical. (A. Jurafsky, Trad.) Buenos Aires: Ricordi Americana

Merton, R. K. (1968). Discussion [of Talcott Parsons, "The position of sociological theory"]. American Sociological Review 13, 164-168.

Musumeci, O. (2002). Hacia una educación de conservatorio humanamente compatible. En I. Martinez y O. Musumesi (Eds.), Actas de la II Reunión Anual de la Sociedad Argentina para las Ciencias Cognitivas de la Música. Quilmes: UNQ. Disponible en: http:// www.saccom.org.ar/ACTAS_CONGRESOS/2do_SACCoM.pdf

Quijano, A. (1992). Colonialidad y modernidad-racionalidad. En H. Bonilla (Ed.) Los conquistados. 1492 y la población indígena de las Américas (pp. 437-447). Bogotá: Tercer Mundo Editores. [Originalmente publicado en 1991 en Perú Indígena, 13 (29)].

Quijano, A. (2002). El regreso al futuro y las cuestiones de conocimiento. En C. Wash, F. Schiwy y S. Castro-Gómez (Eds.), Indisciplinar las ciencias sociales. Geopolíticas del conocimiento y colonialidad del poder. Perspectivas desde lo andino (pp. 45-60). Quito: Abya-YalaUniversidad Andina Simón Bolívar.

Shifres, F., y Burcet, M. I. (2016). La paradoja del canto en el saber musical universitario. Revista de Investigaciones en Técnica Vocal, 4(1), pp. 23-41. Disponible en https://revistas. unlp.edu.ar/RITeV/article/view/2733

Shifres, F. y Gonnet, D. (2015). Problematizando la herencia colonial en la Educación Musical. Epistemus - Revista de estudios en Música, Cognición y Cultura, 3, pp. 51-67. https://doi.org/10.21932/epistemus.3.2971.2

Strauss, A. y Corbin, J. (1990). Basics of qualitative research: Grounded Theory procedures and techniques. London: Sage.

Strauss, A. y Corbin, J. (1994). Grounded theory methodology: an overview. In N.K. Denzin e Y. Lincoln (Eds.), Handbook of qualitative research (pp. 273-285). Thousand Oaks, California: Sage.

Wallerstein, I. [1983] 2006. El capitalismo bistórico. México: Siglo XXI Editores.

Walsh, C. (2007). ¿Son posibles unas ciencias sociales/culturales otras? Reflexiones en torno a las epistemologías decoloniales. Nómadas, 26, 102-113. Disponible en: http:// www.redalyc.org/articulo.oa?id=105115241011 